\title{
əInteraction of Near-Inertial Waves with an Anticyclonic Vortex
}

\author{
Hossein A. KAfiabad, ${ }^{a}$ JACques VAnNeste, ${ }^{\mathrm{a}}$ AND William R. Young ${ }^{\mathrm{b}}$ \\ ${ }^{a}$ School of Mathematics and Maxwell Institute for Mathematical Sciences, University of Edinburgh, \\ Edinburgh, United Kingdom \\ ${ }^{\mathrm{b}}$ Scripps Institution of Oceanography, University of California, San Diego, La Jolla, California
}

(Manuscript received 28 October 2020, in final form 25 March 2021)

\begin{abstract}
Anticyclonic vortices focus and trap near-inertial waves so that near-inertial energy levels are elevated within the vortex core. Some aspects of this process, including the nonlinear modification of the vortex by the wave, are explained by the existence of trapped near-inertial eigenmodes. These vortex eigenmodes are easily excited by an initial wave with horizontal scale much larger than that of the vortex radius. We study this process using a wave-averaged model of near-inertial dynamics and compare its theoretical predictions with numerical solutions of the three-dimensional Boussinesq equations. In the linear approximation, the model predicts the eigenmode frequencies and spatial structures, and a near-inertial wave energy signature that is characterized by an approximately time-periodic, azimuthally invariant pattern. The wave-averaged model represents the nonlinear feedback of the waves on the vortex via a wave-induced contribution to the potential vorticity that is proportional to the Laplacian of the kinetic energy density of the waves. When this is taken into account, the modal frequency is predicted to increase linearly with the energy of the initial excitation. Both linear and nonlinear predictions agree convincingly with the Boussinesq results.
\end{abstract}

KEYWORDS: Inertia-gravity waves; Ocean dynamics; Waves, oceanic; Vortices; Potential vorticity

\section{Introduction}

The trapping of near-inertial waves by anticyclonic axisymmetric vortices is a rare and happy case in which ocean observations (Kunze 1986; Kunze et al. 1995; Kunze and Toole 1997) are in broad agreement with theory (Kunze and Boss 1998; Llewellyn Smith 1999; Danioux et al. 2015) and with numerical models (Lee and Niiler 1998; Zhai et al. 2005). The physical process responsible for wave trapping is that the negative core vorticity extends the internal wave band to frequency slightly below the Coriolis frequency $f$ so that waves with frequency less than $f$ are trapped within the vortex (Kunze 1985).

Anticyclonic near-inertial trapping is readily illustrated with a numerical solution. The top row of Fig. 1 shows a solution of the Boussinesq equations starting from an initial condition consisting of a barotropic vortex superimposed with a large-amplitude wavy disturbance. The vortex has initial Gaussian vertical vorticity

$$
\zeta(x, y, z, 0)=-\operatorname{Ro} f e^{-r^{2} / a^{2}},
$$

where $r=\left(x^{2}+y^{2}\right)^{1 / 2}$ is a radial coordinate, $a$ is the vortex radius, and $f$ is the Coriolis parameter. The Rossby number in (1) is based on the vorticity extremum:

$$
\mathrm{Ro}=\left|\zeta_{\min }\right| / f
$$

¿ Denotes content that is immediately available upon publication as open access.

Corresponding author: Hossein A. Kafiabad, h.kafiabad@ed. ac.uk
The vortex is distorted by a near-inertial wave that is initially horizontally uniform and a plane wave in the vertical, as specified by the initial horizontal velocity

$$
u^{\prime}(x, y, z, 0)+i v^{\prime}(x, y, z, 0)=\phi_{0} e^{i m z},
$$

where $\phi_{0}$ is a constant initial amplitude-see Fig. 1a-and $m$ is a vertical wavenumber. In (3), the primes indicate the nearinertial-wave contribution to the velocity; this is added to the velocity associated with the vorticity $\zeta$ of the axisymmetric vortex in (1). The initial condition has no vertical velocity and no buoyancy perturbations to the uniform buoyancy frequency $N$. If there is no vortex $(\mathrm{Ro}=0)$ then the disturbance in (3) evolves as a horizontally uniform vertical plane wave with $\exp (i m z-i f t)$. The Gaussian vorticity, however, perturbs the effective inertial frequency so that the velocity vectors in Figs. $1 \mathrm{~b}$ and $1 \mathrm{c}$ rotate at different rates. This dephasing is accompanied by a concentration of wave energy into the core of the anticyclonic vortex. For comparison, the lower row of Fig. 1 shows the evolution of the initial disturbance in (3) if the sign of the vorticity in (1) is reversed so that the wave is dephased by a cyclone: wave energy is expelled from the cyclone.

The assumption of a barotropic vortex in (1) and the vertical-plane-wave initial condition in (3) are significant idealizations: real vortices have baroclinic structure and nearinertial waves are forced at the sea surface. Thus there is downward energy propagation, the accumulation of nearinertial energy at the base of the vortex and the formation of critical levels (Joyce et al. 2013; Lelong et al. 2020). Despite the idealizations in (1) and (3), the strong effect of eddy vorticity on near-inertial energy level evident in Fig. 1 occurs in more complicated and realistic flows containing many interacting baroclinic vortices, separated by regions with significant strain (Asselin and Young 2020; Asselin et al. 2020; Thomas et al. 2020). 
(a) $\mathrm{t}=0.00$

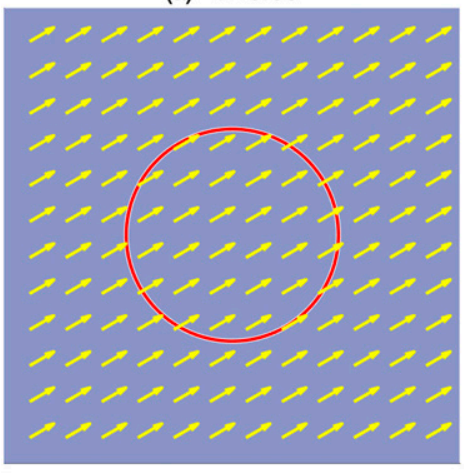

(d) $\mathrm{t}=0.00$

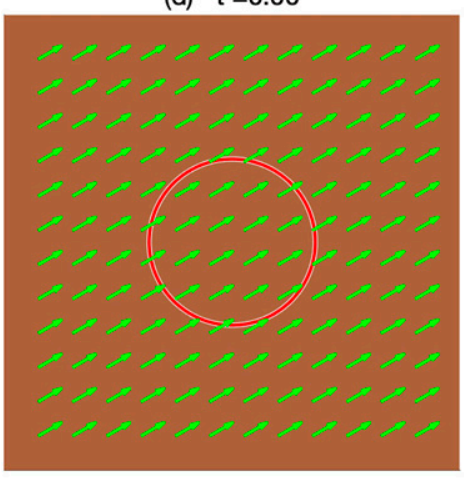

(b) $\mathrm{t}=\mathbf{2 0 . 3 5}$

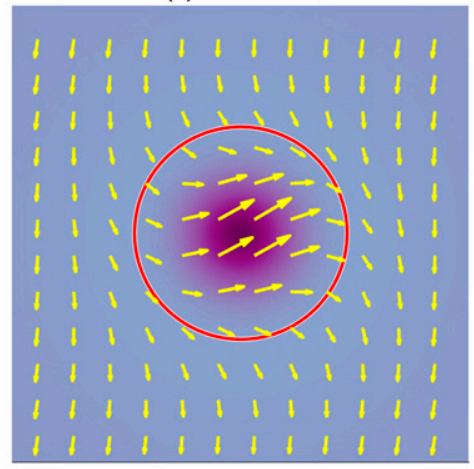

(e) $\mathrm{t}=20.62$

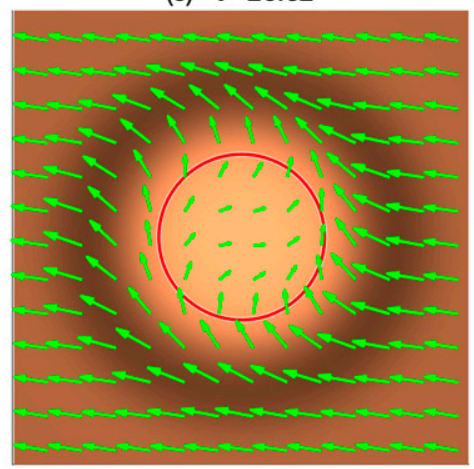

(c) $\mathrm{t}=45.78$

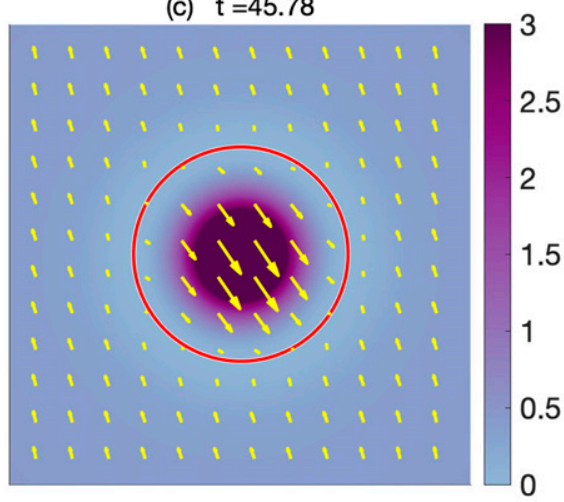

FIG. 1. Visualization of a Boussinesq simulation of near-inertial waves propagating on a Gaussian vortex. This is a slice at $z=0$ showing wave velocity vectors superimposed on the wave kinetic energy density, $\left(u^{\prime 2}+v^{\prime 2}\right) / 2$, indicated by color. The vortex is barotropic, and the wave has a fixed vertical wavenumber $m$. Thus, the location $z$ of the slice does not change the result. The snapshots are taken at times indicated in inertial periods above each panel; the red circle has the vortex radius $a$ in (1). The parameters are those of simulation L13Aa detailed in Table 1 . Shown are (top) the anticyclonic case, $\zeta(r)<0$, and (bottom) the cyclonic case with the same vorticity profile, $\zeta(r)>0$.

Elipot et al. (2010) show that the resulting patterns of sea surface near-inertial activity can be mapped globally using surface drifters.

The main features of the spatial pattern of phase changes in the top row of Fig. 1, and the concentration of wave energy into the anticyclonic core, can be understood by linearizing the Boussinesq equations around a basic state consisting of an anticyclonic barotropic vortex, for example the Gaussian vortex in (1), and then solving an eigenvalue problem to obtain the trapped near-inertial modes of the vortex (Kunze et al. 1995; Kunze and Boss 1998). Instead of linearizing the Boussinesq equations, Llewellyn Smith (1999) used the phase-averaged equation of Young and Ben Jelloul (1997, hereinafter YBJ) to show how the spatially uniform initial wave in (3) excites the linear eigenmodes of the vortex. The details of this linear eigenproblem are, however, not without controversy and novelty: some authors argue that the lowest frequency of the internal wave band is $f+\zeta_{\min }$ (Kunze and Boss 1998; Joyce et al. 2013), while others maintain it is $f+\zeta_{\min } / 2$ (Llewellyn Smith 1999; Chavanne et al. 2012). We have more to say about this issue later: we show that the lowest possible frequency of the trapped eigenmode is $f+\zeta_{\min } / 2$.
The top row of Fig. 1 shows that despite the azimuthal symmetry of the base-state vortex, the trapped eigenmode is not a radial pulsation for which the wave velocity would have a dominant radial component. Observations of trapped nearinertial disturbances in a warm-core ring describe a similar structure: see Fig. 14 of Kunze et al. (1995) and the associated discussion. Describing the phase of the back-rotated velocity $\left(u^{\prime}+i v^{\prime}\right) \exp (i f t)$, Kunze et al. (1995) stress the "lack of horizontal phase progression in the ring core"; this uniformity of phase within the vortex core is a good approximation in Fig. 1b and is strikingly appropriate in Fig. 1c.

Further details of the initial value problem are shown in Fig. 2. In the anticyclonic case (top row) the initially uniform wave kinetic energy, $\left(u^{\prime 2}+v^{\prime 2}\right) / 2$, localizes inside the vortex core and then spreads radially to reform an almost horizontally uniform field. This cycle of radial contraction and expansion, also evident in the time series in Fig. 3, repeats with a period that is much longer than the inertial period. This subinertial oscillation is a signature of the vortex eigenmode and is the topic of this paper. We contrast this periodic behavior with that obtained in a cyclonic vortex, illustrated by the bottom rows of Figs. 1 and 2. In the cyclonic case, wave 
(a) $\mathrm{t}=0.00$

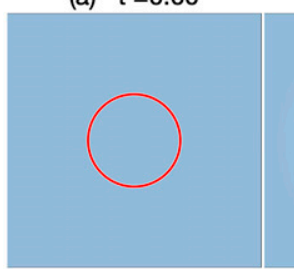

(b) $\mathrm{t}=20.35$

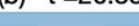

(c) $t=43.24$

(d) $t=76.30$

(e) $\mathrm{t}=99.19$

(f) $t=122.09$

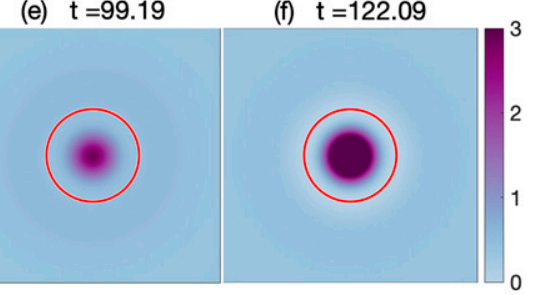

(g) $\mathrm{t}=0.00$

(h) $t=9.52$

(i) $\mathrm{t}=20.62$

(j) $t=31.72$

(k) $t=42.82$

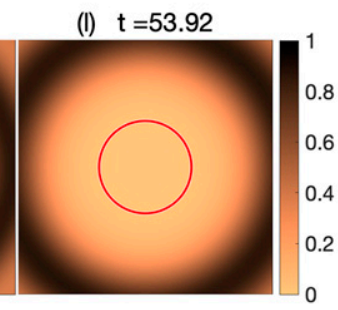

FIG. 2. Horizontal slices of wave kinetic energy $\left(u^{\prime 2}+v^{\prime 2}\right) / 2$ for the same simulation as in Fig. 1. Snapshots are taken at times indicated in inertial periods above each panel. Shown are (top) the anticyclonic case, $\zeta(r)<0$, and (bottom) the cyclonic case, $\zeta(r)>0$.

kinetic energy is expelled from the vortex core, creating a void that expands outwards in time; there is no subinertial pulsation of wave energy.

This paper has two main aims. First, we assess how the predictions for the dynamics of trapped modes made by Llewellyn Smith (1999) using linear theory and the YBJ model apply to nonlinear three-dimensional Boussinesq simulations. Second, we examine how nonlinear effects, specifically those associated with wave-induced changes in the vortex, impact this dynamics.

We start by formulating the vortex eigenmode problem in the YBJ approximation, focusing on the mode with azimuthally uniform backrotated velocity observed in Figs. 1 and 2 (section 2). We add to Llewellyn Smith's (1999) analysis by (i) deriving an approximation for the modal frequency in the limit of small frequency corresponding to weakly trapped modes, which gives us a handle on the number of branches of the dispersion relation, and (ii) showing that the lowest accessible frequency is $f+\zeta_{\min } / 2$.
We compare the theoretical predictions of the eigenmode problem with a series of high-resolution Boussinesq simulations (section 3 ) covering a broad range of parameters, finding an excellent agreement in spite of the complexities introduced by the excitation of a continuous spectrum of (nontrapped) modes, finite Rossby and Burger numbers, finite domain size, and nonlinearity. We consider the effect of weak nonlinearity in section 4: using the nonlinear, phase-averaged model of Xie and Vanneste (2015), in which the YBJ equation is coupled to a quasigeostrophic model, we predict a nonlinear frequency shift that increases the period of trapped mode, and we test this prediction against Boussinesq simulations. This quantitative comparison is a significant test of the phase-averaged model and essential in developing confidence in its accuracy in more complicated situations, such as the propagation of near-inertial waves through geostrophic turbulence characterized by a population of coherent almost axisymmetric vortices (Rocha et al. 2018; Asselin and Young 2020).
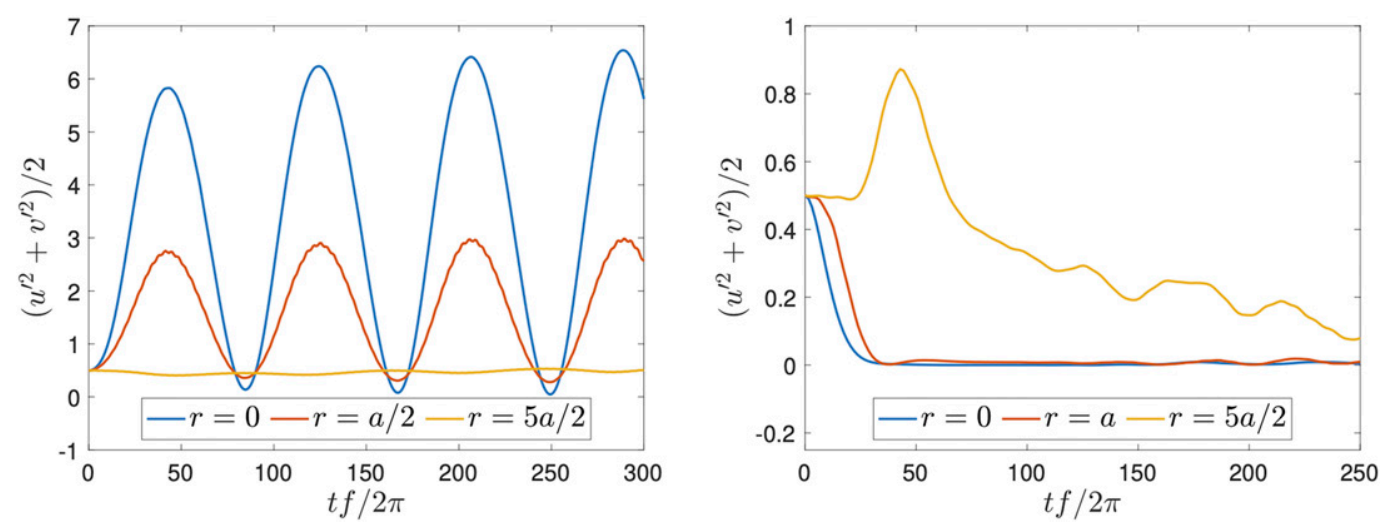

FIG. 3. Time series of the wave kinetic energy at the three points given in the legend for (left) an anticyclone and (right) a cyclone. The vortex center is at $r=0$. For the anticyclone, the parameters are those of simulation L13Ba detailed in Table 1, and for the cyclone they are those of simulation L13Aa with positive sign of vorticity. 


\section{Eigenproblem for the anticyclonic vortex}

\section{a. YBJ vortex eigenmode problem}

Following Llewellyn Smith (1999) we use the YBJ phaseaveraged description of subinertial evolution to solve the vortex eigenmode problem. This assumes a weak vortex, with Ro $\ll 1$, and near-inertial wave frequencies. Other authors have approached this same problem by linearization of the full Boussinesq equations of motion (Kunze et al. 1995; Kunze and Boss 1998). This direct assault leads to an intricate eigenproblem that reduces to the simpler YBJ eigenproblem in the relevant limit.

For the vertical-plane wave initial condition (3), the master variable used in the YBJ equation is the back-rotated velocity

$$
\phi(x, y, t)=\left[u^{\prime}(x, y, z, t)+i v^{\prime}(x, y, z, t)\right] e^{i(f t-m z)},
$$

where $u^{\prime}$ and $v^{\prime}$ are the horizontal wave velocities. Because the vortex is barotropic, and because the waves have the special initial condition in (3), the back-rotated velocity $\phi$ is independent of $z$. To a good approximation the Boussinesq solutions also have this simple structure. This enables convenient separation of the wave quantities from the balanced flow: the balanced component of the solutions is obtained by a vertical average. The remaining baroclinic part of any field is a good approximation for the wave part of that field.

Using (4), the YBJ model can be simplified for barotropic flows and constant buoyancy frequency $N$ to

$$
\frac{\partial \phi}{\partial t}+J(\psi, \phi)+\frac{i}{2} \zeta \phi=\frac{i}{2} \hbar \Delta \phi
$$

where $\Delta=\partial_{x}^{2}+\partial_{y}^{2}$ is the horizontal Laplacian. The second and third terms in (5) are advection by the streamfunction $\psi$ and refraction by the vorticity $\zeta=\Delta \psi$ of the balanced flow. In the dispersive term on the right-hand side of (5),

$$
\hbar \stackrel{\text { def }}{=} N^{2} /\left(f m^{2}\right)
$$

is the dispersivity of near-inertial waves with vertical wavenumber $m$ [see Danioux et al. (2015) for further discussion on this parameter].

Following Llewellyn Smith (1999), we look for eigensolutions of (5) in the form of

$$
\phi(r, \theta, t)=A(\eta) e^{i(\nu \theta-\omega t)},
$$

where $\eta=r / a=\left(x^{2}+y^{2}\right)^{1 / 2} / a$ is a nondimensional radial coordinate, $\theta$ is the azimuthal angle, $\nu=0,1, \ldots$ is the azimuthal wavenumber, and $\omega$ is the frequency of the eigenmode. Introducing (7) into (5) and using the Gaussian form (1) of the vortex leads to

$$
A_{\eta \eta}+\frac{1}{\eta} A_{\eta}+\lambda\left(e^{-\eta^{2}}+\nu \frac{1-e^{-\eta^{2}}}{\eta^{2}}\right) A-\left(\sigma+\frac{\nu^{2}}{\eta^{2}}\right) A=0
$$

where

$$
\sigma=-\frac{2 a^{2} \omega}{\hbar}>0
$$

is a convenient nondimensional frequency. In (8), the strength of the vortex is characterized by the ratio of the vortex angular momentum to the wave dispersivity

$$
\lambda=\frac{a^{2}\left|\zeta_{\min }\right|}{\hbar}
$$

Introducing the Burger number

$$
\mathrm{Bu}=\left(\frac{N}{f m a}\right)^{2}=\frac{\hbar}{f a^{2}},
$$

the vortex-strength parameter can be rewritten as the ratio

$$
\lambda=\mathrm{Ro} / \mathrm{Bu} \text {. }
$$

The YBJ model assumes that $\lambda$ is fixed as $\mathrm{Ro}$ and $\mathrm{Bu} \rightarrow 0$.

To ensure that the mode decays exponentially at great distances from the vortex center, the frequency $\sigma$ in (9) must be positive so that

$$
A \sim e^{-\sqrt{\sigma} \eta} \rightarrow 0, \text { as } \eta \rightarrow \infty .
$$

The other boundary condition defining the eigenproblem for $\sigma$ and $A$ is that the mode has no singularity at $\eta=0$, which is equivalent to $A^{\prime}(0)=0$.

\section{b. Azimuthal wavenumber $\nu=0$}

In the remainder of the paper, we focus on modes with $\nu=0$, which reduces the eigenproblem (8) to

$$
\frac{d^{2} A}{d \eta^{2}}+\frac{1}{\eta} \frac{d A}{d \eta}+\left(\lambda e^{-\eta^{2}}-\sigma\right) A=0 .
$$

There are several reasons for considering only $\nu=0$. Llewellyn Smith (1999) showed that trapped modes with $\nu<0$ do not exist. And, after a vain numerical search for modes with $\nu>0$, he concluded that "we do not know if such solutions exist, nor can we prove that they do not exist." We are pleased to ignore this open problem because the Boussinesq solution in the top row of Figs. 1 and 2 shows that the initial condition in Fig. 1a excites only $\nu=0$ modes. With $\nu=0$, the eigenproblem in (14) is the same as Schrödinger's equation with an axisymmetric Gaussian potential.

The absence of modes with nonzero $\nu$ in Figs. 1 and 2 is remarkable because the initial condition breaks azimuthal symmetry by selecting a special direction: all the velocity vectors in Fig. 1a point northeast. Despite this broken azimuthal symmetry, the trapped disturbance is axisymmetric in the sense ${ }^{1}$ that (i) velocity vectors lying on any circle of radius $r$ in top row of Fig. 1a are identical to one another and (ii) the kinetic energy density in Fig. 2 is axisymmetric. As discussed in section 1, this is consistent with the structure observed by Kunze et al. (1995) in a warm-core ring.

In the Boussinesq eigenproblem of Kunze et al. (1995) and Kunze and Boss (1998), the master variable is the radial

\footnotetext{
${ }^{1}$ The vector field $(u, v)$ is not axisymmetric in the usual sense, that is, $\theta$ independent and pointing in the radial direction.
} 
component of velocity $u_{r}(r, \theta, t)$. The relation $u_{r}+i u_{\theta}=(u+$ $i v) e^{-i \theta}$ then shows that a $\theta$-independent backrotated velocity $\phi$, that is, our $\nu=0$, corresponds to the azimuthal wavenumber $n=-1$ of Kunze and Boss (1998). While use of the backrotated velocity $\phi$ in a problem with axial symmetry might at first sight seem unnatural, the simplicity of the YBJ equation and associated eigenproblem shows its effectiveness in examining near-inertial waves in small-Rossby-number flows; see Llewellyn Smith (1999) for a detailed discussion.

\section{c. Solution of the eigenproblem (14)}

We now turn to solution of the boundary-value problem in (13) and (14). Asymptotic calculations detailed in appendix A show that for all values of $\lambda$, including very weak vortices with $\lambda \ll 1$, there is at least one trapped mode. We refer to this important solution as the zeroth mode and denote its corresponding eigenfunction and eigenvalue by $A_{0}$ and $\sigma_{0}$, respectively: numerical results in Fig. 4 illustrate the form of the zeroth-mode solution. The eigenproblem in (14) is analogous to the quantum mechanical problem of trapping in an axisymmetric Gaussian potential well; in that context the zeroth mode is known as the ground state of the well.

As $\lambda$ increases, additional trapped modes appear through a sequence of bifurcations arising at $\lambda=\lambda_{n}, n=1,2, \ldots$ Figure 5 shows the first two eigenbranches, $\sigma_{0}(\lambda)$ and $\sigma_{1}(\lambda)$. The structure of the corresponding eigenfunctions is illustrated in Fig. 6, which shows $A_{0}(\eta)$ and $A_{1}(\eta)$ for $\lambda=25$.

The bifurcations giving rise to new branches of the dispersion relation can be analyzed by solving the eigenvalue problem in the asymptotic limit $\sigma \rightarrow 0$ corresponding to weakly trapped modes: see (13). This is done in appendix A, where we find that the first three branches arise for

$$
\left\{\lambda_{0}, \lambda_{1}, \lambda_{2}\right\}=\{0,11.1, \ldots, 35.1, \ldots\} .
$$

(The second mode is off stage in Fig. 5.) On each branch, $\sigma \rightarrow 0$ very rapidly as $\lambda \rightarrow \lambda_{n}$ : our analysis shows that

$$
\sigma_{n} \propto \exp \left(-\frac{d_{n}}{\lambda-\lambda_{n}}\right), \quad \text { as } \lambda \downarrow \lambda_{n},
$$

where the $d_{n}$ are constants that can be evaluated explicitly. For the zeroth mode, $\lambda_{0}=0$ and (16) reduces to

$$
\sigma_{0} \sim \exp \left[2\left(\ln 2-\gamma_{\mathrm{E}}\right)-4 / \lambda\right] \quad \text { as } \quad \lambda \downarrow 0,
$$

where $\gamma_{\mathrm{E}}$ is the Euler-Mascheroni constant. The exponential sensitivity of $\sigma_{n}(\lambda)$ to $\left(\lambda-\lambda_{n}\right)^{-1}$ explains the very flat curves as $\lambda \downarrow \lambda_{n}$ in Fig. 5. In Fig. 7 we verify the asymptotic prediction (16) for $n=0$ and 1 by comparison with the numerical solutions of the eigenproblem (14).

In the Boussinesq numerical solution the initial condition in (3) will project onto all of the trapped eigenmodes of the Gaussian vortex in (1). The vortex used for illustrative purposes in Figs. $1-3$ has $\lambda=13.1$. Because

$$
11.1<13.1<35.1
$$

this vortex has two trapped modes (the zeroth and first). Only the zeroth mode is evident in Figs. 1-3, however,

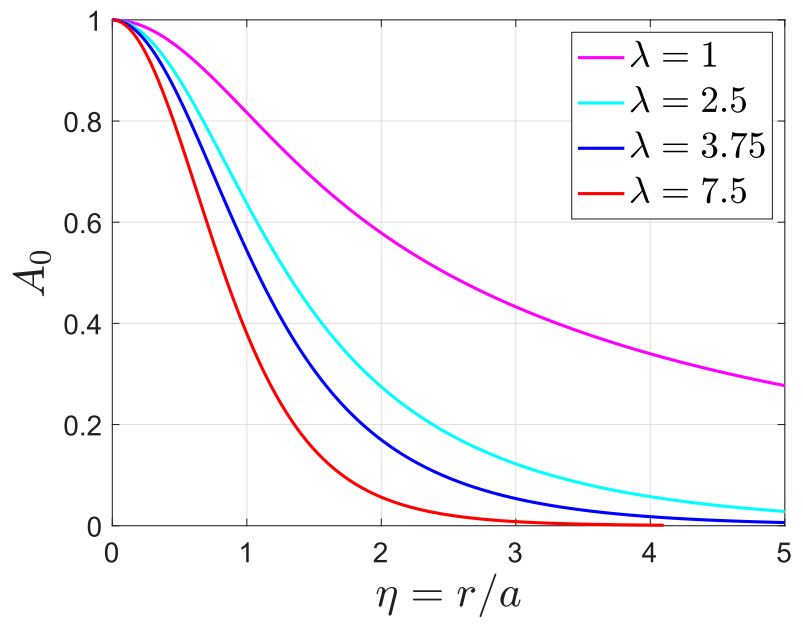

FIG. 4. Eigenfunctions corresponding to the largest eigenvalue $\sigma_{0}$ for different $\lambda$. This is the zeroth eigenmode, which is characterized by having no zeros. As the vortex strength $\lambda$ increases, the mode becomes more tightly trapped to the vortex core and its eigenvalue $\sigma_{0}$ increases: see Fig. 5 below for $\lambda$ as a function of $\sigma$.

presumably because the initial condition projects only weakly on the first mode.

\section{d. The lowest vortex-mode frequency}

A bound on the frequency of subinertial oscillations can be obtained from the eigenproblem (14). Untangling the nondimensionalization, the total frequency of the eigenmode in dimensional variables is

$$
f+\operatorname{Bu} f \omega=f\left(1-\frac{1}{2} \operatorname{Bu} \sigma\right)=f+\frac{1}{2} \frac{\sigma}{\lambda} \zeta_{\min } .
$$

For the $\nu=0$ modes studied here, the issue of whether the lowest frequency of the internal wave band is $f+\zeta_{\min }$ or $f+$ $\zeta_{\min } / 2$ devolves to whether the ratio $\sigma / \lambda$ in (19) is ever greater than 1. Examination of Fig. 5 indicates that for the Gaussian vortex $\sigma / \lambda$ is less than 1 and thus for these modes $f+\zeta_{\min } / 2$ is the lowest possible frequency.

We now establish this property for a general compact vortex, with a vorticity profile $\zeta(r)$ satisfying

$$
\zeta(0)=\zeta_{\min } \leq \zeta(r)<0 .
$$

The generalization of the eigenproblem (14) is

$$
\frac{d^{2} A}{d \eta^{2}}+\frac{1}{\eta} \frac{d A}{d \eta}+[\lambda f(\eta)-\sigma] A=0
$$

where $0<f(\eta) \leq 1$ is (minus) the nondimensional vorticity profile. Multiplying by $\eta$, integrating and using the boundary conditions of trapped modes leads to

$$
\frac{\sigma}{\lambda}=\frac{\int_{0}^{\infty} f(\eta) A(\eta) \eta d \eta}{\int_{0}^{\infty} A(\eta) \eta d \eta}
$$




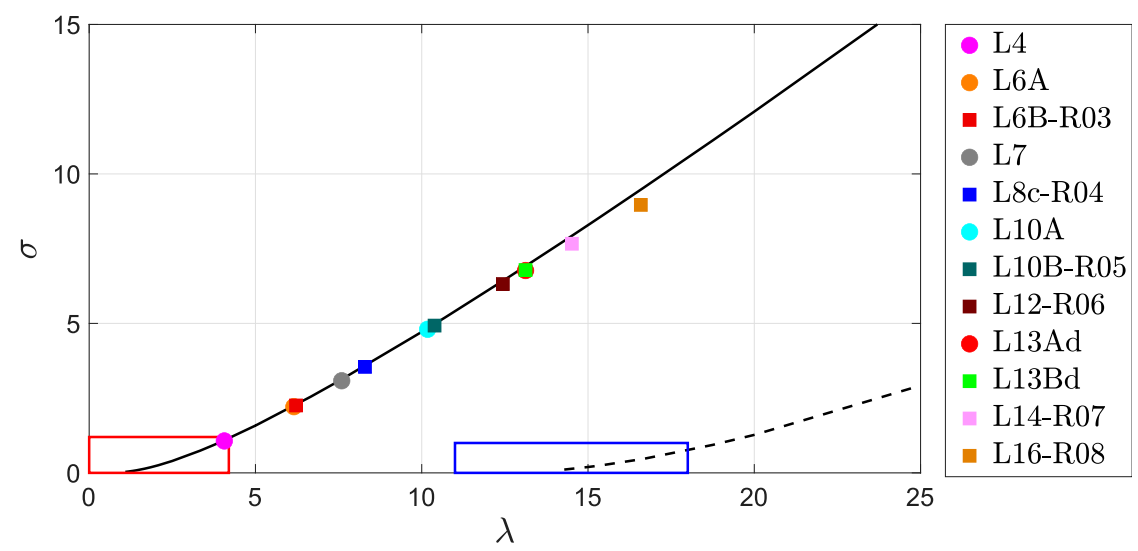

FIG. 5. Nondimensional frequency $\sigma$ of trapped modes for different $\lambda$ derived by numerical solution of the YBJ eigenproblem (14). The two branches shown correspond to the zeroth mode (solid curve) and first mode (dashed curve). The colored symbols correspond to the simulations in Table 1 that are shown in the legend. See Fig. 7 below for a magnified view of the two outlined rectangles.

The zeroth mode, also known as the ground state, has the largest frequency $\sigma$ and a sign-definite eigenfunction; hence

$$
\frac{\sigma_{n}}{\lambda} \leq \frac{\sigma_{0}}{\lambda} \leq \max _{\eta} f(\eta)=1
$$

which completes the argument.

\section{Comparison with numerical solutions of Boussinesq equations}

We now assess the analytical results of previous sections against a suite of high-resolution nonhydrostatic Boussinesq solutions in a triply periodic domain. In these simulations, the flow is initialized with the planar wave in (3) superimposed on the barotropic vortex in (1). To maintain the periodicity of the initial field, the Gaussian vortex in (1) is slightly modified by discretizing it in the Fourier space and truncating the unresolved high-wavenumber modes. A dealiased pseudospectral solver detailed in Kafiabad et al. (2021) is used to derive the numerical solutions, and a third-order Adams-Bashforth scheme is used for time integration. A hyperdissipation of the form $\nu_{h}\left(\partial_{x}^{2}+\partial_{y}^{2}\right)^{4}+\nu_{z} \partial_{z}^{8}$ is used in the momentum and buoyancy equations. The flow parameters and setup are in Table 1 . These parameters are such that the simulations correspond to the interaction of mesoscale vortices with wind-generated waves. The Gaussian vortex in (1) has maximum azimuthal velocity of $0.32 \mathrm{Ro} f a$ at $r=1.13 a$, which is around 1.3 for $\operatorname{Ro}=0.05, f=200$, and $a=0.4$ (typical values listed in Table 1). Considering the values of wave energy densities $E_{0}$, the initial wave velocity ranges between 0.3 and 1 , which makes the wave velocity at the same order or slightly smaller than the vortex maximum velocity. To map this to the ocean context, the value $\mathrm{Ro}=0.05$ is realistic for large-scale flows; the dimensional parameters $f=10^{-4} \mathrm{~s}^{-1}$ and a vortex radius $a=100 \mathrm{~km}$ then give a maximum azimuthal velocity of $0.16 \mathrm{~m} \mathrm{~s}^{-1}$ for the vortex and wave velocities in the range $0.05-0.16 \mathrm{~m} \mathrm{~s}^{-1}$.
The first aspect of the theoretical results that can be compared with the numerical solutions of Boussinesq equations is the frequency of subinertial oscillations such as those observed in Fig. 3 and the top row of Fig. 2. For each simulation of Table 1 , we estimate the scaled frequency, $\sigma_{\text {sim }}$, defined in (9) by averaging the times between consecutive troughs and peaks of wave energy at $r=0$. We also solve (14) for the value of $\lambda$ in each simulation to derive the zeroth eigenfrequency $\sigma_{0}$. In the last column of Table 1 , the normalized difference between $\sigma_{\text {sim }}$ and $\sigma_{0}$ is shown. Within the range of $\lambda \leq 13$, this difference remains less than $2 \%$, if the simulations with the lowest wave energy level for each set of parameters are considered. This remarkable agreement is shown in Fig. 5 by superimposing $\sigma_{\text {sim }}$

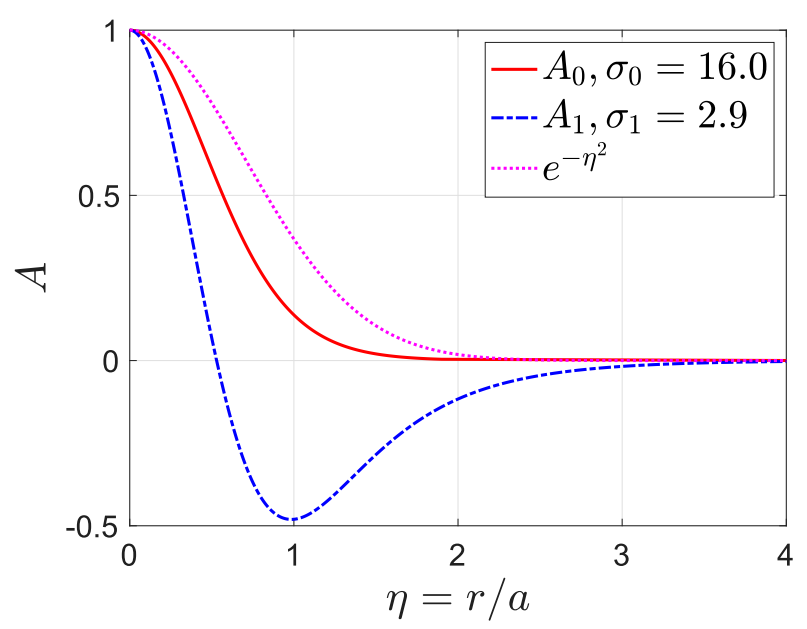

FIG. 6. Two eigenmodes of the Gaussian vortex with $\lambda=25$ with their corresponding eigenvalues in the legend. The Gaussian ex$\mathrm{p}\left(-\eta^{2}\right)$ is shown as guidance. The zeroth mode corresponds to $\sigma_{0}=16.0$; the first mode, with $\sigma_{1}=2.9$ and one zero at $\eta=0.53$, is more weakly trapped than the zeroth mode. 

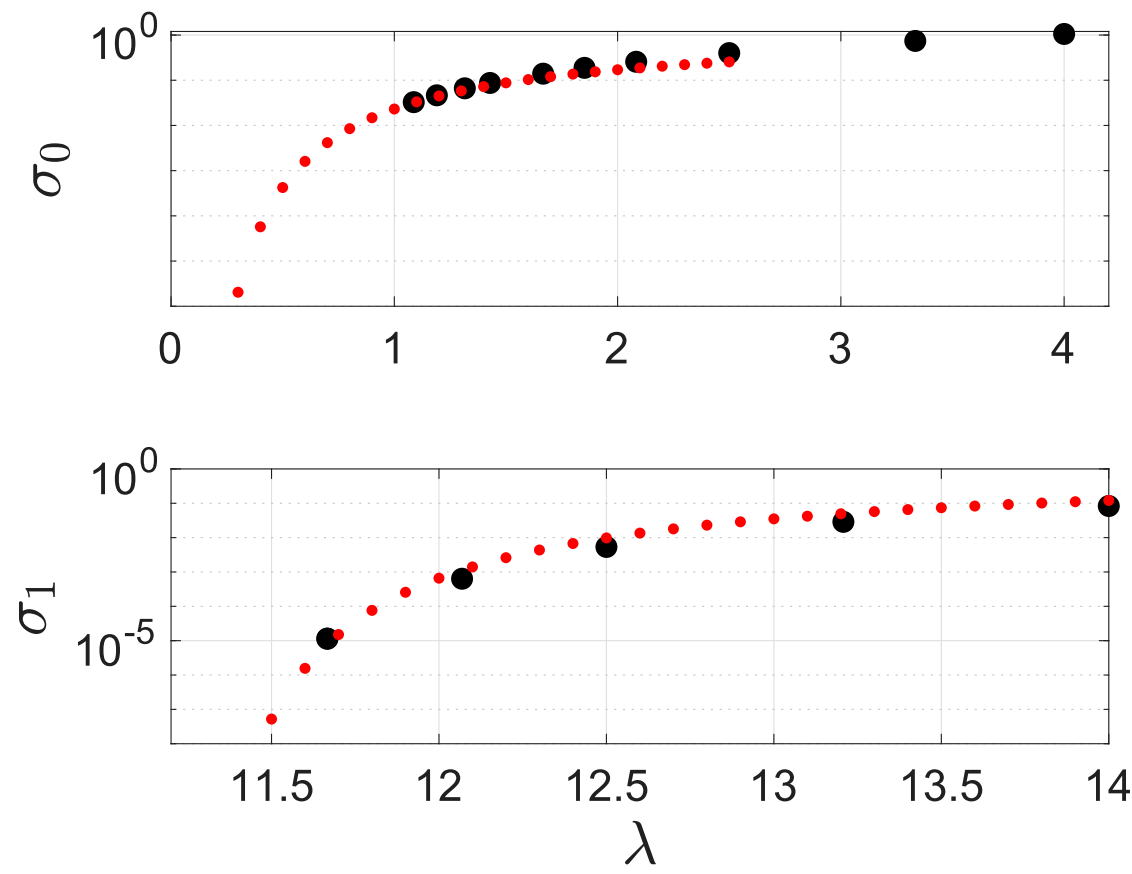

FIG. 7. Magnified view of the (top) red-outlined rectangle and (bottom) blue-outlined rectangle from Fig. 5, with logarithmic vertical axis. Numerical results (large black dots) are compared with the asymptotic results in (16) and (17) (small red dots).

on the zeroth eigenbranch. The colored symbols in this figure correspond to those in the second last column of Table 1. For $\lambda>13$, the projection of initial condition on the first eigenvector (in addition to the zeroth one) affects the slow modulation of wave energy observed in the simulations: this first mode component increases the relative difference between $\sigma_{\text {sim }}$ and $\sigma_{0}$.

The simulations with the same parameters, but increasing initial wave energy, reveal a systematic dependence of the modal frequency on the amplitude of the initial wave. This

TABLE 1. List of Boussinesq simulations with their corresponding parameter values. All simulations share the following parameters: horizontal domain size $L=2 \pi$, vertical domain size $H=2 \pi / 36$, number of grid points on the $x$ and $y$ axes $N_{x}=N_{y}=1152$, number of grid points on the $z$ axis $N_{z}=96$, horizontal hyperviscosity $\nu_{h}=5 \times 10^{-18}$, and vertical hyperviscosity $\nu_{\mathrm{z}}=5 \times 10^{-23}$. The domain size, $2 \pi \times$ $2 \pi$, determines the unit of length. The unit of time is defined such that the nondimensional Coriolis parameter is $f=200$; hence for a dimensional $f=10^{-4} \mathrm{~s}^{-1}$, the unit of time is $200 \times 10^{4} \mathrm{~s}=23.15$ days.

\begin{tabular}{lcccccccccc}
\hline \hline Simulation & $N$ & $a$ & $m$ & \multicolumn{1}{c}{$\Delta t$} & $E_{0}$ & Ro & Bu & $\lambda$ & $\sigma_{\text {sim }}$ & $\left|\sigma_{0}-\sigma_{\text {sim }}\right| / \sigma_{0}$ \\
\hline L4 & 1600 & 0.28 & 288 & $1.74 \times 10^{-4}$ & 0.1 & 0.04 & 0.0098 & 4.06 & 1.07 & $1.93 \%$ \\
L6A & 1300 & 0.28 & 288 & $1.74 \times 10^{-4}$ & 0.05 & 0.04 & 0.0065 & 6.16 & 2.21 & $1.28 \%$ \\
L6B-R03 & 1600 & 0.40 & 288 & $1.74 \times 10^{-4}$ & 0.05 & 0.03 & 0.0048 & 6.22 & 2.26 & $1.17 \%$ \\
L7 & 1600 & 0.50 & 180 & $1.74 \times 10^{-4}$ & 0.1 & 0.06 & 0.0079 & 7.59 & 3.08 & $1.48 \%$ \\
L8a & 960 & 0.40 & 288 & $1.30 \times 10^{-4}$ & 0.5 & 0.04 & 0.0048 & 8.29 & 3.45 & $3.57 \%$ \\
L8c-R04 & 1600 & 0.40 & 288 & $1.74 \times 10^{-4}$ & 0.05 & 0.04 & 0.0048 & 8.29 & 3.55 & $0.90 \%$ \\
L10A & 1300 & 0.36 & 288 & $1.56 \times 10^{-4}$ & 0.1 & 0.04 & 0.0039 & 10.18 & 4.80 & $0.69 \%$ \\
L10B-R05 & 1600 & 0.4 & 288 & $1.74 \times 10^{-4}$ & 0.05 & 0.05 & 0.0048 & 10.37 & 4.93 & $0.82 \%$ \\
L10C & 1600 & 0.45 & 216 & $1.74 \times 10^{-4}$ & 0.1 & 0.07 & 0.0068 & 10.33 & 4.87 & $1.40 \%$ \\
L12-R06 & 1600 & 0.4 & 288 & $1.74 \times 10^{-4}$ & 0.05 & 0.06 & 0.0048 & 12.44 & 6.32 & $1.66 \%$ \\
L13Aa & 1600 & 0.45 & 288 & $1.30 \times 10^{-4}$ & 0.5 & 0.05 & 0.0038 & 13.12 & 6.58 & $4.88 \%$ \\
L13Ab & 1600 & 0.45 & 288 & $1.30 \times 10^{-4}$ & 0.2 & 0.05 & 0.0038 & 13.12 & 6.71 & $3.00 \%$ \\
L13Ac & 1600 & 0.45 & 288 & $1.30 \times 10^{-4}$ & 0.1 & 0.05 & 0.0038 & 13.12 & 6.76 & $2.27 \%$ \\
L13Ad & 1600 & 0.45 & 288 & $1.30 \times 10^{-4}$ & 0.05 & 0.05 & 0.0038 & 13.12 & 6.77 & $2.08 \%$ \\
L13Ba & 960 & 0.27 & 288 & $1.74 \times 10^{-4}$ & 0.5 & 0.05 & 0.0038 & 13.12 & 6.39 & $7.50 \%$ \\
L13Bd & 960 & 0.27 & 288 & $1.74 \times 10^{-4}$ & 0.05 & 0.05 & 0.0038 & 13.12 & 6.78 & $1.85 \%$ \\
L14-R07 & 1600 & 0.4 & 288 & $1.74 \times 10^{-4}$ & 0.05 & 0.07 & 0.0048 & 14.51 & 7.66 & $3.34 \%$ \\
L16-R08 & 1600 & 0.4 & 288 & $1.74 \times 10^{-4}$ & 0.05 & 0.08 & 0.0048 & 16.59 & 8.96 & $5.39 \%$ \\
\hline
\end{tabular}




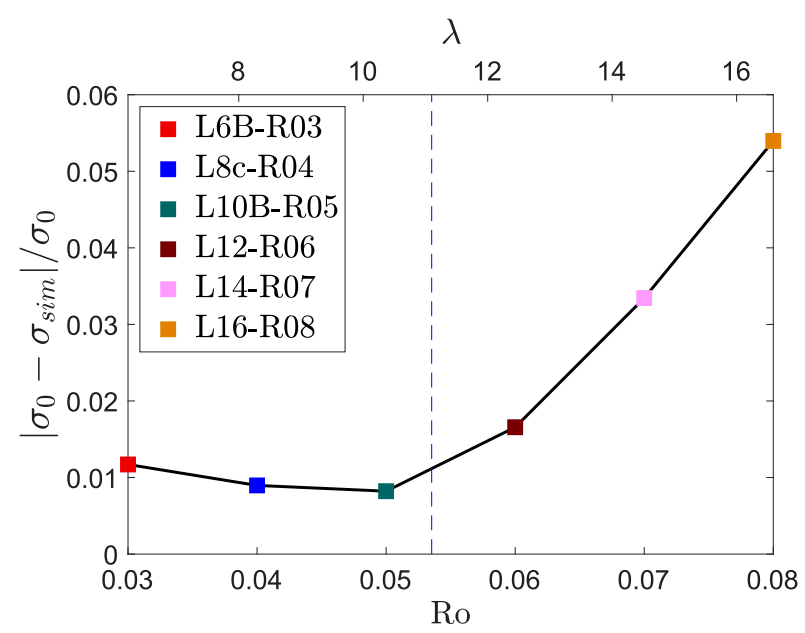

FIG. 8. Relative difference between the modal frequency estimated from Boussinesq simulations and the smallest eigenfrequency of the YBJ model as a function of Ro. The color-coded symbols correspond to the simulations of Table 1 . The second eigenmode exists to the right side of the dashed vertical line marking $\lambda=\lambda_{1}$.

dependence is not captured by the YBJ model, because it neglects the nonlinear wave feedback onto the balanced flow. Analogy with other nonlinear oscillators suggests that this feedback likely results in a frequency shift that depends on the wave energy level. We will discuss this phenomenon in depth in the next section and estimate the frequency shift using the coupled model of Xie and Vanneste (2015). Setting this frequency shift aside, the remaining differences between results based on the YBJ model and the Boussinesq solution can plausibly be attributed to some combination of

(i) inaccuracy in the YBJ equation resulting from the finite Rossby and Burger numbers,

(ii) finite domain size of the Boussinesq code, and

(iii) low-resolution sampling frequency of the times series used to calculate $\sigma_{\text {sim. }}$.

Figure 8 displays $\left|\sigma_{0}-\sigma_{\text {sim }}\right| / \sigma_{0}$ as function of Ro for a suite of simulations with identical parameters, but varying Ro. Increasing Ro increases the discrepancy between $\sigma_{0}$ and $\sigma_{\text {sim }}$. This is partly due to nonlinear effects, not captured in the linear YBJ model-issue $\mathrm{i}$ - and partly due to excitation of the higher eigenmodes that appear as Ro and therefore $\lambda$, is increased. For very small values of $\lambda$, a long integration time is required to capture a few oscillations, which leads to reentering of the waves back to the domain and interactions with the mean flow and other waves-issue ii. Such a long time scales, however, do not have realistic implications in the interaction of oceanic flows with waves. For instance, the eigenperiod of the case $\lambda=3$ is more than 230 inertial periods.

Comparing the eigenfunctions of section $2 \mathrm{c}$ with the simulations is less straightforward, because the initial condition (3) excites not only trapped vortex eigenmodes but also a continuous spectrum (Llewellyn Smith 1999). Taking this into account, the solution of (5) can be written as

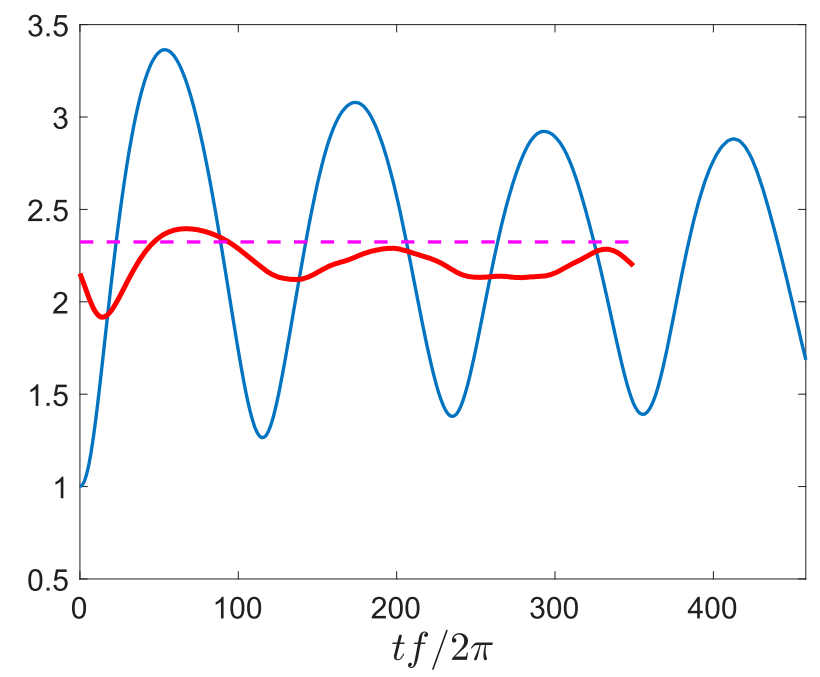

FIG. 9. Wave amplitude $|\phi|$ at $r=0$ (blue curve), back-rotated velocity at $r=0$ after removing the continuum, $\left|\phi-\phi_{\text {cont }}\right|$ (red curve), and $\alpha_{0} \phi_{0}=2.32$ calculated from (27) (dashed magenta) for simulation L8a.

$$
\phi=\phi_{0}\left[\sum_{p=0}^{N-1} \alpha_{p} A_{p}(r / a) e^{-i \omega_{p} t}+\phi_{\text {cont }}(r, \tau)\right],
$$

where $\alpha_{p}$ is the projection of the normalized initial condition onto mode $A_{P}$ and $N=N(\lambda)$ is the number of trapped modes for given $\lambda$. Here we set $N=1$ since we are considering values of $\lambda$ where the higher eigenmodes either do not exist or their eigenfrequency is much lower than $\omega_{0}$. The term $\phi_{\text {cont }}$ is the "continuum remnant" that is left over because the trapped modes do not form a complete basis; Llewellyn Smith (1999) shows that $\phi_{\text {cont }}$ depends logarithmically on time for large time. Because this time dependence is slow when compared with $1 / \omega_{0}$, the continuum remnant can be estimated by integrating over 1 eigenperiod,

$$
\phi_{\text {cont }}(r, t) \approx \frac{\omega_{0}}{2 \pi} \int_{t}^{t+2 \pi / \omega_{0}} \phi(r, s) d s,
$$

and removed from the solution to obtain

$$
\alpha_{0} \phi_{0} A_{0}(r / a)=\phi(r / a, t)-\phi_{\text {cont }}(r / a, t)
$$

$A_{0}$ is orthogonal to all higher modes $A_{p}(p>0)$ and to $\phi_{\text {cont }}$. Hence, after multiplying both sides of (24) by $A_{0} \eta$ and integrating (at $t=0), \alpha_{0}$ is

$$
\alpha_{0}=\frac{\int A_{0}(\eta) \eta d \eta}{\int A_{0}^{2}(\eta) \eta d \eta} .
$$

To investigate the accuracy of (26) we evaluate both sides at $r=0$ for the simulation L8a. We obtain $A_{0}(\eta)$, with normalization $A_{0}(0)=1$, by numerical solution of eigenproblem (14). Using this solution we find that the left-hand side of (26) is $\alpha_{0} \phi_{0}=2.32$, where (27) is used to calculate $\alpha_{0}$; the constant 


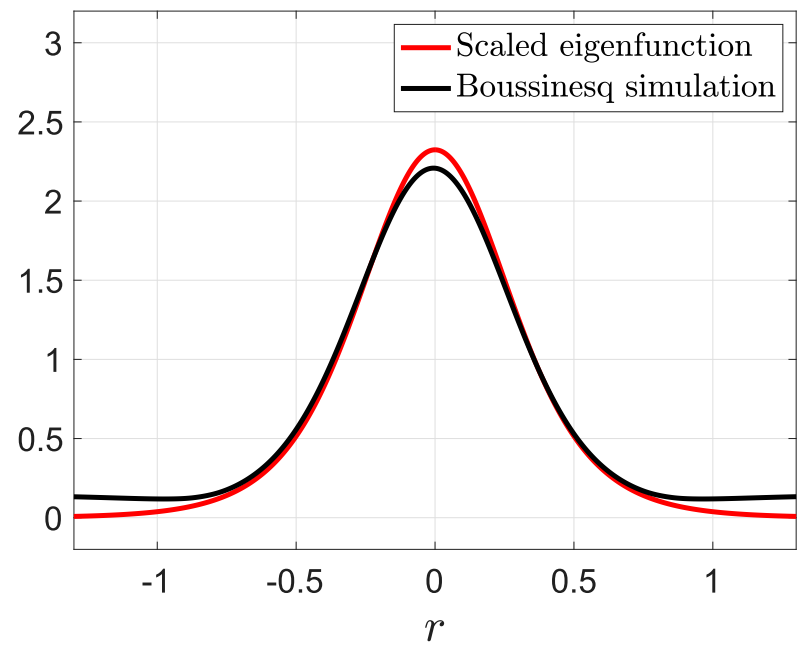

FIG. 10. Scaled eigenfunction $\alpha_{0} \phi_{0} A_{0}(r)$, with $\alpha_{0}$ computed using (27) (red curve) as compared with $\left|\phi(r, t)-\phi_{\text {cont }}(r, t)\right|$ extracted from data from simulation L8a (black curve).

2.32 is the dashed magenta line in Fig. 9. For the right-hand side of (26), the blue sinusoidal curve in Fig. 9 is $|\phi(0, t)|$ computed using the baroclinic velocity fields of the Boussinesq simulation. The right-hand side of (26) is obtained from the Boussinesq solution, resulting in the red curve in Fig. 9. The time average of the red curve is 2.24 , which is close to the prediction $\alpha_{0} \phi_{0}=2.32$.

After gaining confidence in (26), we scale $A_{0}(r / a)$, which is computed by solving (14), by $\alpha_{0} \phi_{0}=2.32$ and compare it with the right-hand side of (26), averaged over 400 inertial periods (about 3 eigenperiods) to remove the small variation in time that was discussed earlier. The results are shown in Fig. 10. Despite many approximations, the agreement between theory and simulation is remarkable. The tails of the two curves, however, display a noticeable difference stemming from the finite-domain effects-point ii above. Repeating the same process for several other simulations of Table 1, we find similar agreement (not shown).

We emphasize that the joint excitation of the zeroth mode and continuous spectrum is necessary to observe the subinertial oscillations of the wave energy displayed in Figs. 2 and 3. Because $\alpha_{0}$ and $A_{0}(r / a)$ are real, exciting solely the zeroth mode results in a time-independent wave energy $\left|\phi_{0}\right|^{2} / 2=\alpha_{0}^{2} A_{0}^{2}(r / a) / 2$.

\section{Nonlinear frequency shift}

According to (14), the oscillation of trapped modes depends only on $\lambda=\mathrm{Ro} / \mathrm{Bu}$. However, after fixing these parameters, we observe that the period of oscillations changes with the initial wave energy $E_{0}=\left|\phi_{0}\right|^{2} / 2$ : see Fig. 11 . To explain this frequency shift we have to go beyond the linear YBJ model. Xie and Vanneste (2015) include the feedback of waves on the time evolution of $\psi$ using a generalized Lagrangian mean (GLM) approach. Wagner and Young (2015) avoid GLM and instead present an alternative derivation using a multitime expansion

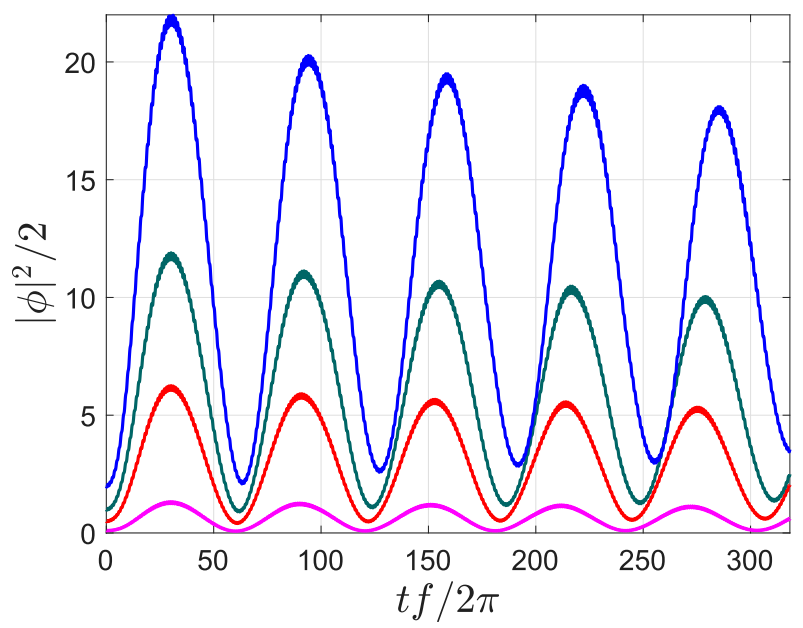

FIG. 11. Time series of wave kinetic energy $|\phi|^{2} / 2$ at the center of the vortex for fixed $\mathrm{Ro}$ and $\mathrm{Bu}$ and varying $E_{0}$. All of the parameters in these simulations are the same as those in L10C, except for $E_{0}$, which is set to 0.1 for the magenta curve, 0.5 for the red curve, 1.0 for the green curve, and 2.0 for the blue curve.

of the Eulerian equations of motion (see also Wagner and Young 2016). The model can succinctly be written for a barotropic flow by adding a nonlinear wave-induced component $q^{W}$ to the linear PV:

$$
q=\Delta \psi+\underbrace{\frac{1}{f}\left[\frac{1}{4} \Delta|\phi|^{2}+\frac{i}{2} J\left(\phi^{*}, \phi\right)\right]}_{q^{W}} .
$$

The material conservation of $q$ together with the YBJ equation (5) form a coupled model for the joint evolution of $q$ and $\phi$, with $\psi$ obtained by inverting the Laplacian in (28) [see Rocha et al. (2018) for the derivation]. We emphasize that $\psi$ is the streamfunction associated with the Lagrangian mean flow; this is crucial for the interpretation of the model, including its energetics (Rocha et al. 2018; Kafiabad et al. 2021).

The model simplifies dramatically when the wave and flow are axisymmetric. The potential vorticity $(28)$ reduces to

$$
\begin{aligned}
q(r) & =\frac{1}{r} \frac{d}{d r}\left(r \frac{d \psi}{d r}\right)+\frac{1}{4 f} \frac{1}{r} \frac{d}{d r}\left(r \frac{d|\phi|^{2}}{d r}\right), \\
& =\Delta\left(\psi+\frac{|\phi|^{2}}{4 f}\right)
\end{aligned}
$$

and its material conservation to the local invariance $\partial_{t} q=0$. For an initially uniform $\phi$, this gives

$$
q=\zeta_{0}=\Delta \psi_{0}=\Delta\left(\psi+\frac{|\phi|^{2}}{4 f}\right),
$$

Kafiabad et al. (2021) confirm the validity of (31) by comparing it with numerical solutions of Boussinesq equations.

Using (31) to eliminate $\zeta=\Delta \psi$ in (5) results in a closed nonlinear equation for $\phi$, 


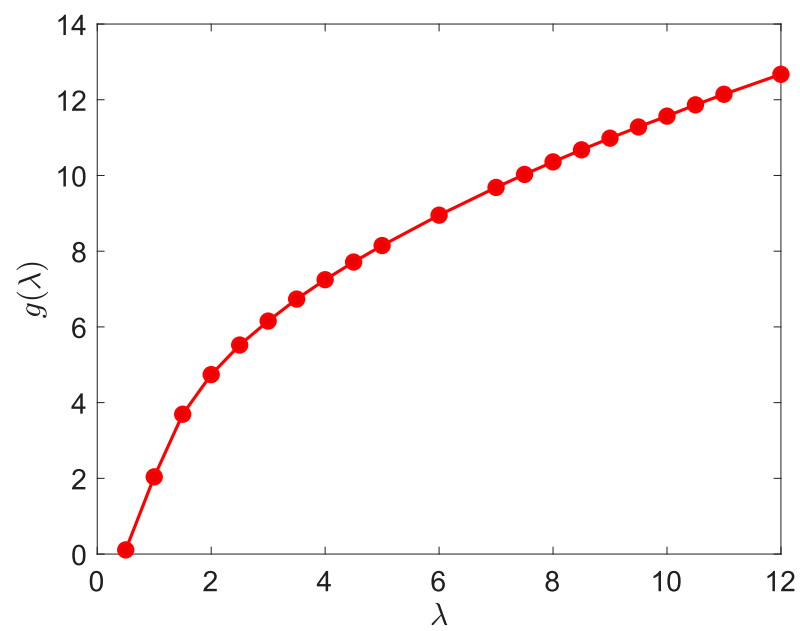

FIG. 12. Numerical evaluation $g(\lambda)$ in (37). The symbols indicate numerical results, and the smooth curve is an interpolant.

$$
\frac{\partial \phi}{\partial t}+\frac{i}{2}\left(\Delta \psi_{0}-\frac{\Delta|\phi|^{2}}{4 f}\right) \phi-\frac{i}{2} \hbar \Delta \phi=0 .
$$

In passing from (5) to (32) there is a significant simplification because $\phi$ is independent of the azimuthal angle so that the advective term $J(\psi, \phi)$ vanishes identically.

We are interested in the weakly nonlinear regime, when the cubic nonlinearity $\Delta|\phi|^{2} \phi /(4 f)$ is small when compared with the linear term $\Delta \psi_{0} \phi$, that is, when $|\phi|^{2} /\left(4 f\left|\zeta_{\min }\right| a^{2}\right) \ll 1$. Based on this small parameter, we solve (32) by introducing the formal parameter $\varepsilon \ll 1$ and rewriting (32) as

$$
\frac{\partial \phi}{\partial t}+\frac{i}{2}\left(\Delta \psi_{0}-\varepsilon \frac{\Delta|\phi|^{2}}{4 f}\right) \phi-\frac{i}{2} \hbar \Delta \phi=0 .
$$

We expand the back-rotated velocity and frequency according to

$$
\begin{aligned}
& \phi=\phi_{0} \alpha_{0} A_{0}(r / a) e^{-i \omega t}+\varepsilon \tilde{\phi}(r, \varepsilon t)+\cdots \quad \text { and } \\
& \omega=\omega_{0}+\varepsilon \tilde{\omega}+\cdots
\end{aligned}
$$

where $\omega_{0}$ and $A_{0}$ are the eigenvalue and eigenfunction of the zeroth trapped mode. Note that the leading-order term in (34) varies on linear time scale $1 / \omega_{0}$ as well as slower time scale $\varepsilon t$, whereas the higher-order terms only vary on the slower time scale. Computations detailed in appendix B lead to the frequency shift

$$
\tilde{\omega}=\frac{\left|\phi_{0}\right|^{2}}{8 f a^{2}} g(\lambda),
$$

where $g(\lambda)$ is the dimensionless function

$$
g(\lambda)=\frac{\left(\int_{0}^{\infty} A_{0} \eta d \eta\right)^{2} \int_{0}^{\infty}\left(\frac{d}{d \eta} A_{0}^{2}\right)^{2} \eta d \eta}{\left(\int_{0}^{\infty} A_{0}^{2} \eta d \eta\right)^{3}}
$$

The frequency shift $\tilde{\omega}$ is therefore quadratic in the wave amplitude $\varphi$; in other words, $\tilde{\omega}$ scales linearly with the wave

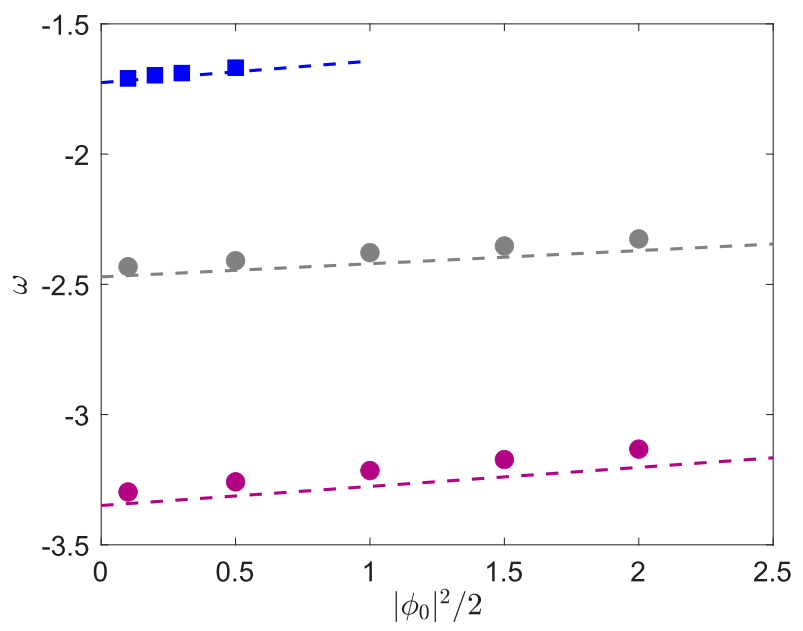

FIG. 13. Subinertial oscillation frequency as a function of initial wave energy: the predicted frequency $\omega=\omega_{0}+\tilde{\omega}$, where $\omega_{0}$ is the linear eigenfrequency and $\tilde{\omega}$ is a shift due to wave feedback; $\tilde{\omega}$ is calculated from (36) (dashed lines) and is compared with numerical estimates (circles and squares) using time series of wave energy at the vortex center. The parameters of simulation L7 (gray), L8c-R04 (blue), and L10C (purple) with varying $E_{0}=\left|\phi_{0}\right|^{2} / 2$ are used.

kinetic energy. The function $g(\lambda)$, computed from the numerical solution of the eigenproblem and shown in Fig. 12, further shows that $\tilde{\omega}$ increases monotonically with $\lambda$; with small $\lambda$ the frequency shift is less significant.

In Fig. 13, the modified eigenfrequency that takes the wave feedback into account, i.e., $\omega=\omega_{0}+\tilde{\omega}$, is compared with the frequency of slow modulations in Boussinesq simulations. Three sets of simulations are considered where all the parameters are fixed within each set while the initial wave energy $\left|\phi_{0}\right|^{2} / 2$ is varied. These results show good agreement between the nonlinear coupled model of Xie and Vanneste (2015) and the Boussinesq simulations and confirm the validity of (36). There is a small offset between the predicted and simulation frequencies of some sets, which is due to the issues i-iii discussed in the previous section. For L8c-R04 (the blue symbols) in Fig. 13 the analysis is limited to $E_{0}<0.05$ : as discussed in the conclusion, for higher amplitude waves the vortex strongly interacts with the near inertial wave.

\section{Conclusions and discussion}

The linearized YBJ model of section 2 focusses attention on the back-rotated velocity-rather than the radial velocity - as the simplest variable characterizing the trapped eigenmodes of an anticyclonic vortex. This is in immediate agreement with solutions of the Boussinesq equations: the top row of Fig. 1 shows that the trapped disturbance does not have a conspicuous radial velocity component. Instead, the back-rotated velocity is approximately independent of azimuth. Thus, the advective term, $J(\psi, \phi)$ in (5), vanishes identically so that near-inertial trapping results only from 
(a) $\mathrm{t}=26.53$

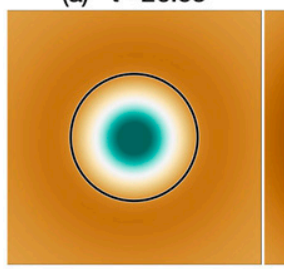

(b) $\mathrm{t}=54.38$

(c) $t=82.23$

(d) $\mathrm{t}=110.08$

(e) $t=137.93$
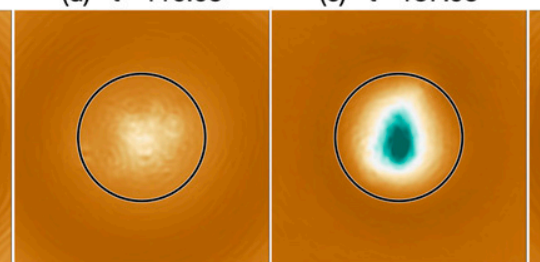

(f) $t=165.79$

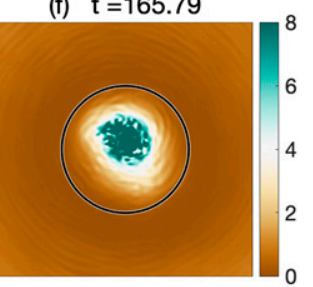

(g) $t=26.53$

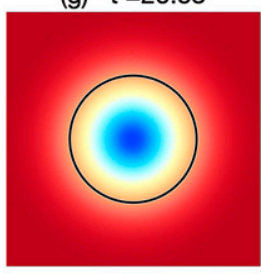

(h) $t=54.38$

(i) $t=82.23$

(j) $t=110.08$

(k) $t=137.93$
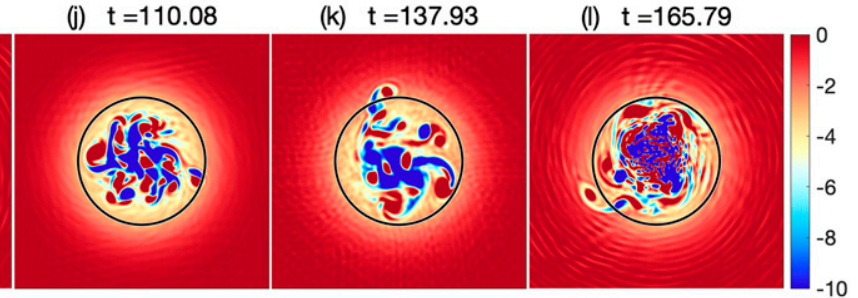

FIG. 14. Horizontal slices of (top) the wave kinetic energy $\left(u^{\prime 2}+v^{\prime 2}\right) / 2$ and (bottom) the barotropic vertical vorticity. All of the parameters in this simulation are the same as those in L8c-R04, except that the initial wave energy is increased by a factor of 30 to $E_{0}=\left|\phi_{0}\right|^{2} / 2=1.5$. Snapshots are taken at the times indicated in inertial periods above each panel.

the $i \zeta \phi / 2$ frequency shift. In section $2 \mathrm{~d}$ we show that, as a consequence, the lowest possible frequency of the vortex eigenmode-the "bottom of the discrete spectrum"-is $f+\zeta_{\min } / 2$.

Exquisite forcing of a single pure eigenmode-designed so as to not excite the continuous spectrum-produces a steady axisymmetric pattern of wave kinetic energy density since $|\phi|^{2}=|A|^{2}$ is then time independent. But generic forcing or initial conditions excites all of the available discrete modes of the vortex, and also a continuum of untrapped disturbances. Thus, in the top row of Fig. 2, we see that the initial condition in (3) - chosen to represent excitation by an atmospheric storm of scale much larger than the vortex scale-results in a low-frequency pulsation of the kinetic energy density, corresponding to the oscillations in the wave kinetic energy time series of Fig. 3. This pulsation is not a single pure eigenmode. In section 3 we extracted the frequency of the subinertial oscillation from Fig. 3 and showed that this modal period is in good agreement with the predictions of the YBJ equation.

Our results provide a detailed picture of the dynamics of near-inertial waves forced at large scales and subsequently trapped by anticyclonic vortices; they should facilitate the identification of such waves from observation and help to explain their behavior. Theoretical work on near-inertial vortex eigenmodes, including results herein, is limited to barotropic vortices (Kunze and Boss 1998; Llewellyn Smith 1999). A future challenge is understanding the near-inertial eigenmodes of baroclinic vortices and the development of critical-layer singularities resulting from the accumulation of near-inertial energy at the base of the vortex. This phenomenon is seen in numerical solutions of both the Boussinesq equations (Lelong et al. 2020) and the phase-averaged coupled model used here (Asselin and Young 2020).

In section 4 we go beyond the linear approximation and test the predictions of the phase-averaged model of Xie and
Vanneste (2015), Wagner and Young (2016), and Rocha et al. (2018). This model couples quasigeostrophic and YBJ models and accounts for the mean-flow change induced by wave feedback (see also Kafiabad et al. 2021) through a wave contributions to PV. We show that the wave feedback leads to frequency shift of the vortex eigenmode that is linearly proportional to the kinetic energy of the eigenmode. We find this frequency shift is in good quantitative agreement with Boussinesq results. This confirms the ability of the phase-averaged coupled model to represent NIW-meanflow interactions.

All results in this work are in the regime of weak nonlinearity. But what happens if one hits the vortex with a very large initial disturbance? Figures 14 and 15 show the result of strongly perturbing a Gaussian vortex by increasing the amplitude of the initial condition $\phi_{0}$ in (3). The initial development of this large disturbance, up to about 55 inertial periods, is similar to that of the weakly nonlinear problem shown in the top row of Fig. 2: the wave kinetic energy concentrates in the vortex core and the barotropic vorticity remains smooth. However, after about 60 inertial periods the core concentration of wave kinetic energy triggers an instability-see Fig. 15. The high-frequency bursts in Fig. 15 are accompanied by the formation of small spatial scales in the vorticity field: in Figs. $14 j-1$, the main anticyclone curdles and small vortex dipoles circulate around its crumbled remains. It is impressive that a prominent subinertial cycle persists and that there are episodes of "relaminarization" coincident with the wave-energy minima in Fig. 15. This low-frequency modulation of the instability is a persistent signature of the vortex eigenmode that survives for over 300 inertial periods. There are open questions about the nature of the instability observed in Fig. 14 that we leave for future work.

Acknowledgments. Authors Kafiabad and Vanneste are supported by the U.K. Natural Environment Research Council 


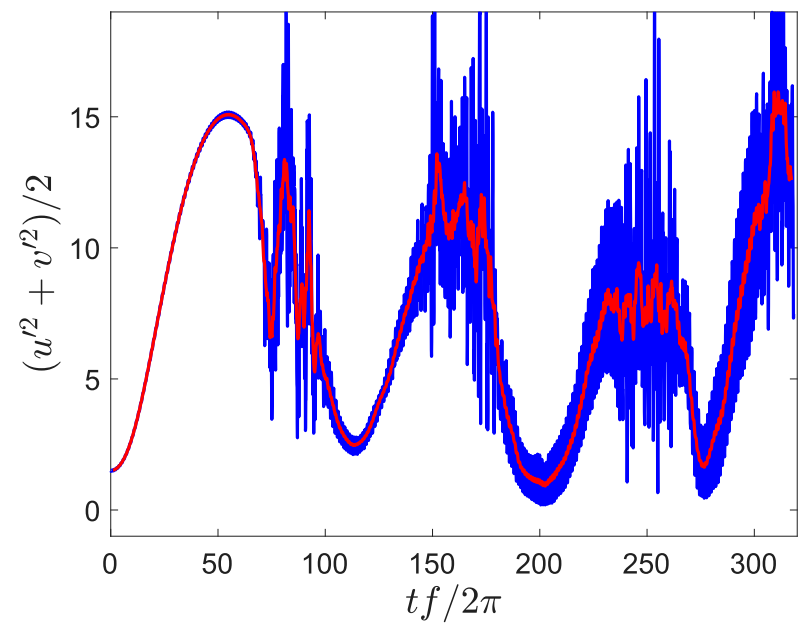

FIG. 15. Time series of wave kinetic energy $\left|\phi_{0}\right|^{2} / 2$ at the center of the vortex for the simulation shown in Fig. 14 in blue and its average over two inertial periods in red.

grant NE/R006652/1. Author Young is supported by the National Science Foundation Award OCE-1657041 and the Office of Naval Research Award N00014-18-1-2803. This work used the ARCHER U.K. National Supercomputing Service.

\section{APPENDIX A}

\section{The Weak-Trapping Limit}

We solve the eigenvalue problem (14) in the weak-trapping regime $\sigma \ll 1$ using matched asymptotics. In the outer region, $\eta \gg 1$, the Gaussian vorticity is exponentially small and can be neglected. This leads to the outer approximation

$$
A(\eta)=q K_{0}(\sqrt{\sigma} \eta)
$$

where $K_{0}$ is the modified Bessel function and $q$ is an undetermined constant. In an intermediate region where $\sqrt{\sigma} \eta \ll 1$ and $\eta \gg 1$, the Bessel function $K_{0}$ is approximated as

$$
A(\eta)=-q \ln \eta-\frac{1}{2} q \ln \sigma+q\left(\ln 2-\gamma_{\mathrm{E}}\right)+\cdots,
$$

where $\gamma_{\mathrm{E}}$ is Euler's constant and we have used the smallargument asymptotics of $K_{0}$.

In the inner region where $\eta=O(1)$, we use $\sigma \ll 1$ to reduce (14) to

$$
\frac{d^{2} A}{d \eta^{2}}+\frac{1}{\eta} \frac{d A}{d \eta}+\lambda e^{-\eta^{2}} A=0
$$

We select the bounded solution as $\eta \rightarrow 0$ by imposing

$$
A(0)=1 \quad \text { and } \quad A^{\prime}(0)=0 .
$$

Equations (A3) and (A4) define an initial-value problem that-except for the zeroth mode in (A9) below-must

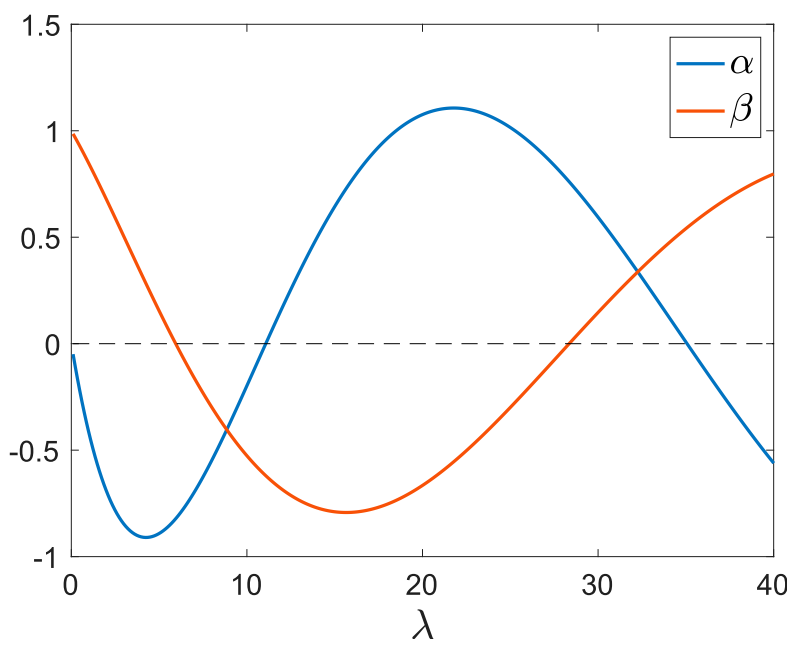

FIG. A1. Functions $\alpha(\lambda)$ and $\beta(\lambda)$ derived by solving (A3) numerically.

be solved numerically. For $\eta \gg 1$, the solution has the asymptotics

$$
A(\eta) \sim \alpha(\lambda) \ln \eta+\beta(\lambda)+O(1),
$$

with $\alpha(\lambda)$ and $\beta(\lambda)$ determined from the numerical solution. Matching (A5) with (A2) results in

$$
\frac{\beta(\lambda)}{\alpha(\lambda)}=\frac{1}{2} \ln \sigma+\gamma_{\mathrm{E}}-\ln 2
$$

This is an equation for $\sigma$ that is valid only for $\beta / \alpha<0$ and $|\beta / \alpha| \gg 1$ so that $\sigma \ll 1$ as assumed. Equation (A6) identifies the zeros $\lambda_{n}, n=0,1, \ldots$ of the function $\alpha(\lambda)$ as the values of $\lambda$ at which new branches of the dispersion relation appear. Note that $\lambda_{0}=0$ corresponds to the zeroth mode; this eigensolution exists even for very weak vortices.

We have computed $\alpha$ and $\beta$ numerically for $0<\lambda \leq 40$ and show the results in Fig. A1. The first bifurcation values of $\lambda$ are found to be $\lambda_{0}=0, \lambda_{1} \approx 11.1$, and $\lambda_{1} \approx 35$.1. In view of the sign of $\beta\left(\lambda_{n}\right)$, new branches appear for $\lambda>\lambda_{n}$. Approximating the left-hand side of (A6) near $\lambda_{n}$ and solving for $\sigma$ leads to the dispersion relations

$$
\sigma \sim \exp \left[2\left(\ln 2-\gamma_{\mathrm{E}}\right)-c_{n} /\left(\lambda-\lambda_{n}\right)\right] \quad \text { as } \quad \lambda \rightarrow \lambda_{n}^{+},
$$

with $c_{n}=-2 \beta\left(\lambda_{n}\right) / \alpha^{\prime}\left(\lambda_{n}\right)>0$.

We can obtain a fully analytic form for the $n=0$ branch (the zeroth mode), with $\lambda \approx \lambda_{0}=0$ by solving (A3) asymptotically for $\lambda \ll 1$. A straightforward expansion in powers of $\lambda$ gives

$$
\begin{aligned}
A(\eta) & =1-\frac{\lambda}{4} \int_{0}^{\eta^{2}} \frac{e^{-x}-1}{x} d x+O\left(\lambda^{2}\right), \\
& =1-\frac{1}{4} \lambda\left[E_{1}\left(\eta^{2}\right)+2 \ln \eta\right]+O\left(\lambda^{2}\right),
\end{aligned}
$$

where $E_{1}$ is the exponential integral. Noting that $E_{1}(\eta) \rightarrow 0$ as $\eta \rightarrow \infty$, we find from (A5) that, as $\lambda \rightarrow 0, \alpha(\lambda) \sim-\lambda / 2$ 
and $\beta(\lambda) \sim 1$; this results in $c_{0}=4$ and the zeroth-mode dispersion relation in (17). For the $n=1$ branch, we find numerically $\alpha^{\prime}\left(\lambda_{1}\right) \approx 0.18$ and $\beta\left(\lambda_{1}\right) \approx-0.62$; hence $c_{1} \approx 6.8$.

\section{APPENDIX B}

\section{Frequency Shift due to Wave Feedback}

Substituting (34) into (33), keeping the terms at order $\varepsilon^{0}$, leads to

$$
-\omega_{0} A_{0}+\frac{1}{2} \Delta \psi_{0} A_{0}-\frac{1}{2} \hbar \Delta A_{0}=0
$$

which is the dimensional form of (14) for $A_{0}$ and $\omega_{0}$. We introduce the self-adjoint operator $\mathscr{L}$,

$$
\mathscr{B}=-\omega_{0}+\frac{1}{2} \Delta \psi_{0}-\frac{1}{2} \hbar \Delta
$$

applied to functions, such as $A_{0}(r)$, that vanish as $r \rightarrow \infty$ and are nonsingular at $r=0$. The terms at the next order form the equation

$$
-\tilde{\omega} A_{0}+\frac{\mathscr{B} \tilde{\phi}}{\alpha_{0} \phi_{0}}-\frac{\alpha_{0}^{2}\left|\phi_{0}\right|^{2}}{8 f} \Delta A_{0}^{2} A_{0}=0,
$$

which can be multiplied by $A_{0}$ and then integrated to obtain

$$
-\tilde{\omega} \int A_{0}^{2} r d r+\int(\mathscr{B} \tilde{A}) A_{0} r d r-\frac{1}{8 f} \int A_{0}^{2} \Delta A_{0}^{2} r d r=0
$$

All integrals run from $r=0$ to $\infty$. Because $\mathscr{L}$ is self-adjoint

$$
\int(\mathscr{L} \tilde{\phi}) A_{0} r d r=\int \tilde{\phi}\left(\mathscr{C} A_{0}\right) r d r=0 .
$$

The last integral in (B4) can also be simplified after integration by parts

$$
\begin{aligned}
\int A_{0}^{2} \Delta A_{0}^{2} r d r & =\int A_{0}^{2} \frac{d}{d r}\left(r \frac{d}{d r} A_{0}^{2}\right) d r \\
& =-\int\left(\frac{d}{d r} A_{0}^{2}\right)^{2} r d r .
\end{aligned}
$$

Using (B5) and (B6), (B4) reduces to the following expression after rewriting the integrals in terms of the dimensionless coordinate $\eta=r / a$

$$
\tilde{\omega}=\frac{\left|\phi_{0}\right|^{2}}{8 f a^{2}} \frac{\left(\int A_{0} \eta d \eta\right)^{2} \int\left(\frac{d}{d \eta} A_{0}^{2}\right)^{2} \eta d \eta}{\left(\int A_{0}^{2} \eta d \eta\right)^{3}},
$$

where we used (27) to substitute for $\alpha_{0}$.

\section{REFERENCES}

Asselin, O., and W. R. Young, 2020: Penetration of windgenerated near-inertial waves into a turbulent ocean.
J. Phys. Oceanogr., 50, 1699-1716, https://doi.org/10.1175/ JPO-D-19-0319.1.

, L. N. Thomas, W. R. Young, and L. Rainville, 2020: Refraction and straining of near-inertial waves by barotropic eddies. J. Phys. Oceanogr., 50, 3439-3454, https://doi.org/ 10.1175/JPO-D-20-0109.1.

Chavanne, C. P., E. Firing, and F. Ascani, 2012: Inertial oscillations in geostrophic flow: Is the inertial frequency shifted by $\zeta / 2$ or by $\zeta$ ? J. Phys. Oceanogr., 42, 884-888, https://doi.org/10.1175/ JPO-D-12-031.1.

Danioux, E., J. Vanneste, and O. Bühler, 2015: On the concentration of near-inertial waves in anticyclones. J. Fluid Mech., 773, R2, https://doi.org/10.1017/jfm.2015.252.

Elipot, S., R. Lumpkin, and G. Prieto, 2010: Modification of inertial oscillations by the mesoscale eddy field. J. Geophys. Res., 115, C09010, https://doi.org/10.1029/2009JC005679.

Joyce, T. M., J. M. Toole, P. Klein, and L. N. Thomas, 2013: A nearinertial mode observed within a Gulf Stream warm-core ring. J. Geophys. Res. Oceans, 118, 1797-1806, https://doi.org/ 10.1002/jgrc.20141.

Kafiabad, H. A., J. Vanneste, and W. R. Young, 2021: Waveaveraged balance: A simple example. J. Fluid Mech., 911, R1, https://doi.org/10.1017/jfm.2020.1032.

Kunze, E., 1985: Near-inertial wave propagation in geostrophic shear. J. Phys. Oceanogr., 15, 544-565, https://doi.org/10.1175/ 1520-0485(1985)015<0544:NIWPIG > 2.0.CO;2.

- 1986: The mean and near-inertial velocity fields in a warmcore ring. J. Phys. Oceanogr., 16, 1444-1461, https://doi.org/ 10.1175/1520-0485(1986)016<1444:TMANIV>2.0.CO;2.

_ and J. M. Toole, 1997: Tidally driven vorticity, diurnal shear, and turbulence atop Fieberling Seamount. J. Phys. Oceanogr., 27, 2663-2693, https://doi.org/10.1175/1520-0485(1997) 027<2663:TDVDSA $>2.0 . \mathrm{CO} ; 2$.

- - and E. Boss, 1998: A model for vortex-trapped internal waves. J. Phys. Oceanogr., 28, 2104-2115, https://doi.org/ 10.1175/1520-0485(1998)028<2104:AMFVTI >2.0.CO;2.

— R. W. Schmitt, and J. M. Toole, 1995: The energy balance in a warm-core ring's near-inertial critical layer. J. Phys. Oceanogr., 25, 942-957, https://doi.org/10.1175/1520-0485(1995)025<0942: TEBIAW $>2.0 . \mathrm{CO} ; 2$.

Lee, D.-K., and P. P. Niiler, 1998: The inertial chimney: The nearinertial energy drainage from the ocean surface to the deep layer. J. Geophys. Res., 103, 7579-7591, https://doi.org/10.1029/ 97JC03200.

Lelong, M.-P., Y. Cuypers, and P. Bouruet-Aubertot, 2020: Nearinertial energy propagation inside a Mediterranean anticyclonic eddy. J. Phys. Oceanogr., 50, 2271-2288, https://doi.org/ 10.1175/JPO-D-19-0211.1.

Llewellyn Smith, S. G., 1999: Near-inertial oscillations of a barotropic vortex: Trapped modes and time evolution. J. Phys. Oceanogr., 29, 747-761, https://doi.org/10.1175/ 1520-0485(1999)029<0747:NIOOAB > 2.0.CO;2.

Rocha, C. B., G. L. Wagner, and W. R. Young, 2018: Stimulated generation: Extraction of energy from balanced flow by nearinertial waves. J. Fluid Mech., 847, 417-451, https://doi.org/ 10.1017/jfm.2018.308.

Thomas, L. N., L. Rainville, O. Asselin, W. R. Young, J. Girton, C. B. Whalen, L. Centurioni, and V. Hormann, 2020: Direct observations of near-inertial wave $\zeta$-refraction in a dipole vortex. Geophys. Res. Lett., 47, e2020GL090375, https:// doi.org/10.1029/2020GL090375.

Wagner, G., and W. R. Young, 2015: Available potential vorticity and wave-averaged quasi-geostrophic flow. 
J. Fluid Mech., 785, 401-424, https://doi.org/10.1017/ jfm.2015.626.

Wagner, G. L., and W. R. Young, 2016: A three-component model for the coupled evolution of near-inertial waves, quasi-geostrophic flow and the near-inertial second harmonic. J. Fluid Mech., 802, 806-837, https://doi.org/10.1017/ jfm.2016.487.

Xie, J.-H., and J. Vanneste, 2015: A generalised-Lagrangian-mean model of the interactions between near-inertial waves and mean flow. J. Fluid Mech., 774, 143-169, https://doi.org/ 10.1017/jfm.2015.251.

Young, W. R., and M. Ben Jelloul, 1997: Propagation of nearinertial oscillations through a geostrophic flow. J. Mar. Res., 55, 735-766, https://doi.org/10.1357/0022240973224283.

Zhai, X., R. J. Greatbatch, and J. Zhao, 2005: Enhanced vertical propagation of storm-induced near-inertial energy in an eddying ocean channel model. Geophys. Res. Lett., 32, L18602, https://doi.org/10.1029/2005GL023643. 